\title{
How to predict allergic contact dermatitis accompanied \\ in patients with atopic dermatitis
}

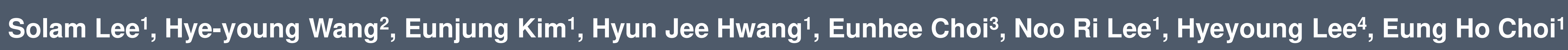

1. Department of Dermatology, Yonsei University Wonju College of Medicine, Wonju, Korea, 2. M\&D, Inc., Wonju Eco Environmental Technology Center, Wonju, Korea
3. Institute of Lifestyle Medicine, Yonsei University Wonju College of Medicine, Wonju, Korea, 4. Department of Biomedical Laboratory Science, Yonsei University, Wonju, Korea

\section{INTRODUCTION}

In patients with atopic dermatitis (AD), the penetration of contact allergens might be potentially increased through disrupted barrier, and hence the risk of sensitization can be also increased. Therefore, there is increasing need for screening of concurrent allergic contact dermatitis (ACD) in AD patients through patch testing.

\section{PURPOSE}

- To examine the proportion of accompanied allergic contact dermatitis (ACD) among the patients with $A D$

To identify the clinical characteristics and genetic variations who are more likely to develop concurrent allergic contact dermatitis in the patients with atopic dermatitis

\section{METHODS AND MATERIALS}

- Study population: 281 patients with AD who underwent patch test

The clinical characteristics and genetic variations were compared between two groups - "AD with ACD": Subjects with any positive result with patch test - "AD only": Subjects with negative patch test result

- Clinical characteristics: Sex, age, onset, duration, type (intrinsic/extrinsic AD), self-reported (family) history of atopic diseases and ACD recalcitrant $A D$, associated skin findings

- Laboratory findings: Total IgE, allergen-specific IgE for DF and DP

- Genetic analysis: FLG 3321delA, K4022X, KLK7, SPINK5, DEFB1, KDR, IL5RA, IL-9, IL12RB1

\section{RESULTS}

Table 1. Comparisons of clinical characteristics between "AD only" and "AD with ACD"

\begin{tabular}{|c|c|c|c|c|c|c|}
\hline & $\begin{array}{l}A D \text { only } \\
(N=210)\end{array}$ & $\begin{array}{c}\text { AD with ACD } \\
(\mathrm{N}=711)\end{array}$ & OR (95\% Cl) & p & $\begin{array}{c}\text { Adjusted OR } \\
(95 \% \mathrm{Cl})^{\mathrm{a}}\end{array}$ & Adjusted $\mathrm{p}^{\mathrm{a}}$ \\
\hline \multicolumn{7}{|l|}{$\overline{\text { Sex }}$} \\
\hline -Male & $136(64.8 \%)$ & $30(42.3 \%)$ & Reference & & & \\
\hline -Female & $74(35.2 \%)$ & $41(57.7 \%)$ & $2.51(1.46-4.38)$ & $<0.001$ & & \\
\hline Age (years) & $13.6 \pm 13.9$ & $27.4 \pm 18.2$ & $1.05(1.03-1.07)$ & $<0.001$ & & \\
\hline Onset (years) & $7.7 \pm 13.0$ & $20.4 \pm 19.4$ & 1.05 (1.03-1.07) & $<0.001$ & $1.01(0.97-1.05)$ & $\pi / 4$ \\
\hline Duration (months) & $70.1 \pm 83.1$ & $82.7 \pm 100.0$ & & 0.338 & & \\
\hline \multicolumn{7}{|l|}{ Type of atopic derma } \\
\hline - Intrinsic & $45(21.4 \%)$ & $7(9.9 \%)$ & Reference & & Reference & \\
\hline - Extrinsic & 165 (78.6\%) & $64(90.1 \%)$ & $2.49(1.13-6.631)$ & 0.046 & $1.53(0.64-4.15)$ & 0.365 \\
\hline Self-reported history of atopic diseases & $109(51.9 \%)$ & $38(53.5 \%)$ & $1.07(0.62-1.84)$ & 0.922 & $1.22(0.67-2.25)$ & 0.521 \\
\hline Self-reported family history of atopic diseases & $164(78.1 \%)$ & 46 (64.8\%) & $0.52(0.29-0.93)$ & 0.038 & $0.91(0.44-1.95)$ & 0.808 \\
\hline Self-reported history of allergic contact derma & $21(10.0 \%)$ & $24(33.8 \%)$ & $4.60(2.36-9.93)$ & $<0.001$ & $1.76(0.80-3.79)$ & 0.153 \\
\hline Self-reported family history of allergic contact & $19(9.0 \%)$ & $22(31.0 \%)$ & $4.51(2.27-9.08)$ & $<0.001$ & $7.09(3.15-16.25)$ & $<0.001$ \\
\hline \multirow{2}{*}{\multicolumn{7}{|c|}{ Associated skin findings }} \\
\hline & & & & & & \\
\hline Impetigo & $20(9.5 \%)$ & $6(8.5 \%)$ & $0.88(0.31-2.16)$ & 0.788 & $1.13(0.35-3.18)$ & \\
\hline Prurigo nodularis & $19(9.0 \%)$ & $15(21.1 \%)$ & $2.69(1.27-5.63)$ & 0.009 & $1.67(0.70-3.87)$ & 0.235 \\
\hline Nummular ecze & $25(11.9 \%)$ & $14(19.7 \%)$ & $1.82(0.87-3.69)$ & 0.103 & $1.02(0.42-2.35)$ & 0.970 \\
\hline Eczema herpeti & $12(5.7 \%)$ & $3(4.2 \%)$ & $0.73(0.16-2.37)$ & 0.631 & 0.41(0.08-1.53) & $\begin{array}{l}{ }^{21} \\
\end{array}$ \\
\hline \multicolumn{7}{|l|}{ Serum IgE level (IU/mL) } \\
\hline $\begin{array}{l}\text { Allallge } \\
\text { Allegen-specific lg E for Dermatooh }\end{array}$ & $\begin{array}{r}04.6 \pm 816.2 \\
30.4 \pm 82.4\end{array}$ & $\begin{array}{r}688.3 \pm \pm 087.5 \\
210.9 \pm 1182.5\end{array}$ & $\begin{array}{l}1.000(1.000-1.00) \\
1.00)\end{array}$ & $\begin{array}{l}0.579 \\
0.977\end{array}$ & $\begin{array}{ll}1.00(1.00-1.100) \\
1.00 \\
(1.00-1.00)\end{array}$ & $\begin{array}{l}0.544 \\
0.927\end{array}$ \\
\hline Allergen-specific IgE for Dermatophagoides pte & $19.2 \pm 39.5$ & $95.7 \pm 498.0$ & $1.00(1.00-1.00)$ & 0.505 & $1.00(1.00-1.00)$ & 0.417 \\
\hline
\end{tabular}

\section{Adjusted by age and sex}

The risk of concurrent $A D$ with $A C D$

$\begin{array}{llllll}\text { Female }>\text { Male } & \text { OR } 2.51 & p<0.001 & \text { Self-reported history of ACD } & \text { OR 4.60 } & p<0.001 \\ \text { Older age } & \text { OR 1.05/year } & p<0.001 & \text { Self-reported family Hx of ACD } & \text { OR 4.51 } & p<0.001 \\ \text { Later onset } & \text { OR 1.05 /year } & p<0.001 & \text { Prurigo nodularis } & \text { OR } 2.69 & p=0.009\end{array}$

- The independent risk after adjusting for age and sex

Self-reported family Hx of ACD $\quad$ OR 7.90 $\quad \mathrm{p}<0.00$

Table 2. Frequency of positive patch-test reactions in the atopic dermatitis patients with concurrent allergic contact dermatitis

\begin{tabular}{|c|c|c|c|}
\hline Allergen & $A D$ with $A C D(n=71)$ & Allergen & AD with ACD $(n=71)$ \\
\hline Cobalt(II) chloride hexahydrate & $27(38.02 \%)$ & Methylisothizzolinone/Methylchloroisothizazolinone & $3\left(4.222^{\circ}\right.$ \\
\hline Nickel(II) sulfate hexahydrate & $18(25.35 \%)$ & Thiuram Mix & \\
\hline Thimerosal & $13(18.30 \%)$ & Colophonium & \\
\hline Mercury(II) amido chloride & $10(14.08 \%)$ & Imidazolidiny Irea & $2(2.819$ \\
\hline p-phenylenediamine & $9(12.67 \%)$ & Myroxylon pereirae resin & $2(2.81 \%$ \\
\hline Potassium dichromate & $8(11.26 \%)$ & Budesonide & $2(2.810$ \\
\hline 4-tetra-butylphenol-formaldehyde resin & $8(11.26 \%)$ & Quaternium-15 & $2(2.810$ \\
\hline Captan & $7(9.85 \%)$ & 2-Mercaptobenzothiazole & $2(2.81 \%$ \\
\hline Formaldehyde & $7(9.85 \%)$ & Trixocortol-21-pivalate & $2(2.810$ \\
\hline Fragrance Mix I & $5(7.04 \%)$ & Lanolin Alcohol (Wool alcohol) & $1(1.40 \%$ \\
\hline Neomycin sulfate & $3(4.22 \%)$ & N-Isopropyl-N-phenyl-4-phenylendiamine & \\
\hline Paraben Mix & $3(4.22 \%)$ & Mercapto Mix & \\
\hline Methylisothiazolinone/Methylchloroisothiazolinone & $3(4.22 \%))>0$ & Clinguinol & \\
\hline
\end{tabular}

The most common sensitized-allergen was cobalt (38.02\%) and nickel (25.35\%) in the AD patients with concurrent ACD

Figure 1. ROC curve by age for predicting concurrent allergic contact dermatitis in the atopic dermatitis patients without self-reported family history of allergic contact dermatitis

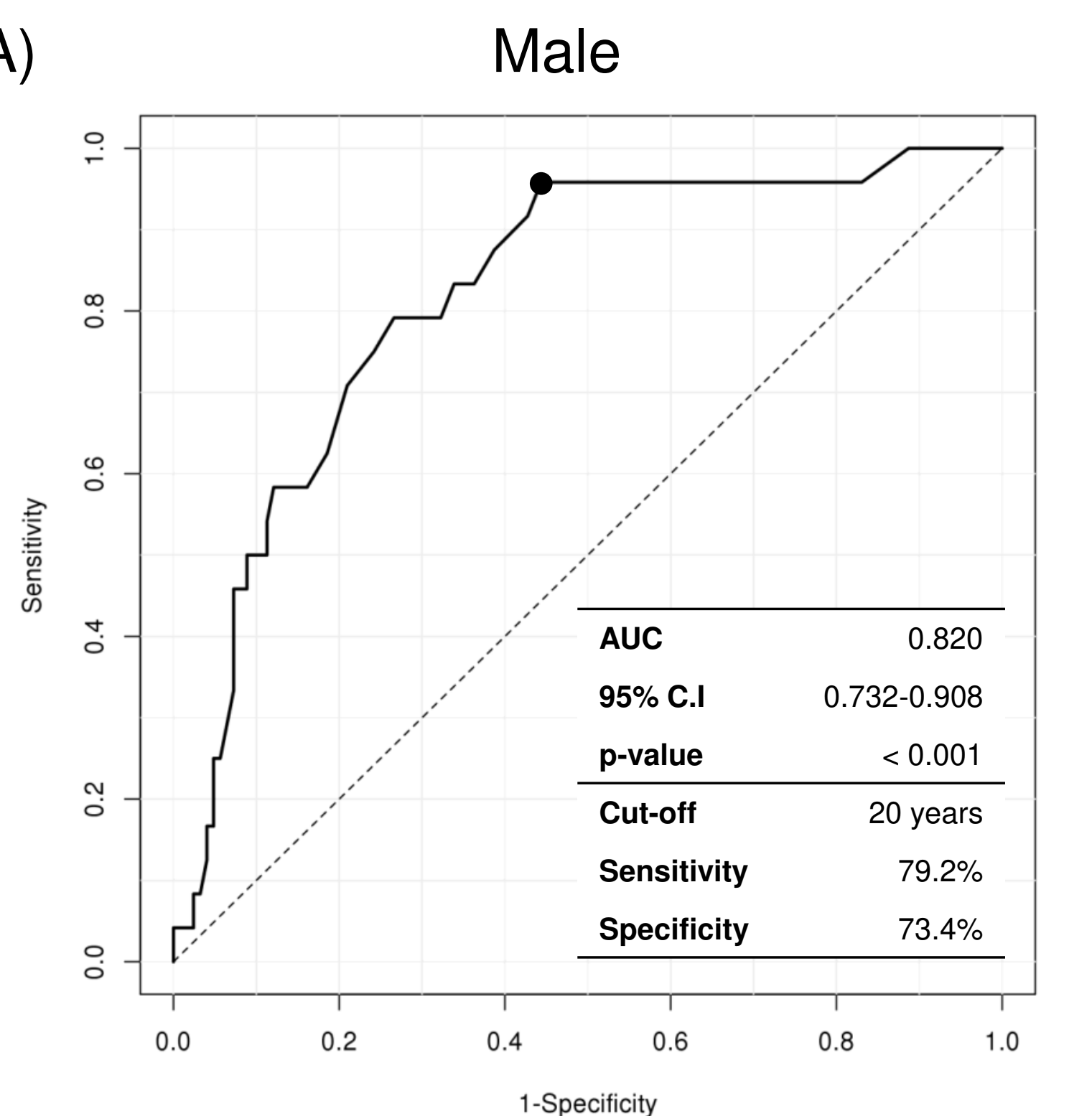

(B)

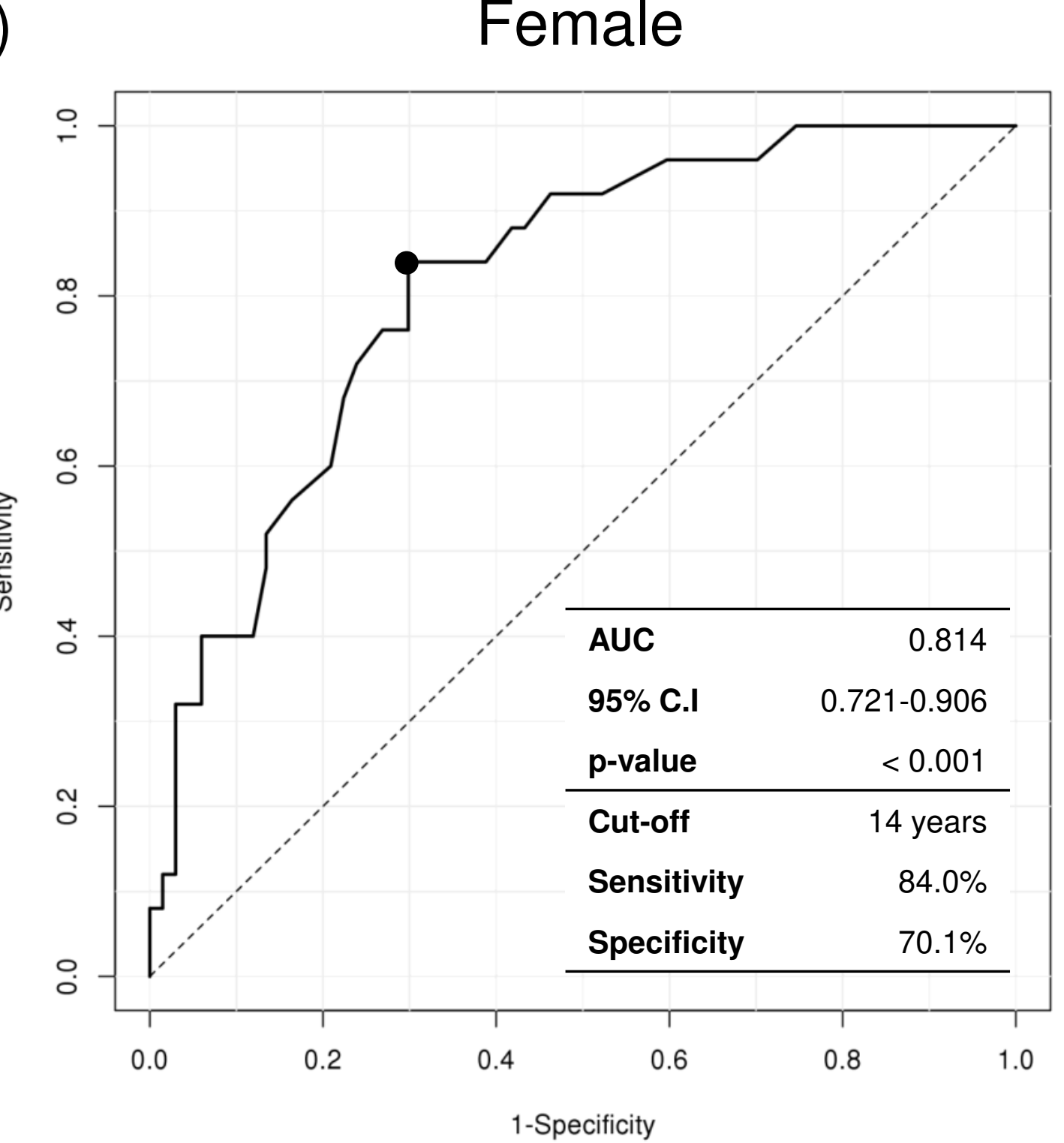

- When to determine to have perform patch testing in the AD patients without self-reported family history of ACD,

"Age" was very useful for predicting concurrent ACD in those patient. (AUC 0.820 in male, AUC 0.814 in female patients) If patch testing is performed to male patients over 20 years old,

concurrent ACD will be diagnosed with sensitivity of $79.2 \%$ and specificity of $73.4 \%$

If patch testing is performed to female patients over 14 years old

concurrent ACD will be diagnosed with sensitivity of $84.0 \%$ and specificity of $70.1 \%$
Table 3. Comparisons of genetic variations between "AD only" and "AD with $A C D$ "

\begin{tabular}{|c|c|c|c|c|c|c|}
\hline & $\begin{array}{l}\text { AD only } \\
(\mathrm{N}=210)\end{array}$ & $\begin{array}{l}\text { AD with ACD } \\
(\mathrm{N}=71)\end{array}$ & OR $(95 \% \mathrm{Cl})$ & $\mathrm{p}$ & $\begin{array}{l}\text { Adjusted OR } \\
\left.(95 \%)^{\circ}\right)^{a}\end{array}$ & Adjusted $p^{a}$ \\
\hline \multirow{2}{*}{\multicolumn{7}{|c|}{$\begin{array}{l}\angle L K 7 \\
-W T\end{array}$}} \\
\hline & & & & & & \\
\hline - Heterozygous MT & $113(53.8 \%)$ & $35(49.3 \%)$ & $0.83(0.46-1.49)$ & 0.520 & 0.75 (0.39-1.44) & 0.381 \\
\hline \multicolumn{7}{|l|}{ SPINK5G1156A } \\
\hline -WT & $191(576 \%)$ & 44 157 7001$)$ & Reterence & & Reference & \\
\hline - Heterozygous MT & $81(38.6 \%)$ & $28(39.4 \%)$ & $1.02(0.58-1.77)$ & 0.944 & $1.00(0.53-1.86)$ & 0.992 \\
\hline - Homozygous MT & $8(3.8 \%)$ & $2(2.8 \%)$ & $0.74(0.11-3.09)$ & 0.708 & $1.09(0.13-5.98)$ & 0.925 \\
\hline \multicolumn{7}{|l|}{ SPINK5 C1188T } \\
\hline - WT & $61(29.0 \%)$ & $23(32.4 \%)$ & Reference & & Reference & \\
\hline - Heterozygous MT & $102(48.6 \%)$ & $33(46.5 \%)$ & $0.86(0.46-1.61)$ & 0.628 & $0.70(0.35-1.41)$ & 0.316 \\
\hline \multicolumn{7}{|l|}{$\begin{array}{l}\text { - Homozygous MT } \\
\text { SPINK5 G2475T }\end{array}$} \\
\hline -WT & $104(49.5 \%)$ & $33(46.5 \%)$ & Reference & & Reference & \\
\hline - Heterozygous MT & $78(37.1 \%)$ & $31(43.7 \%)$ & $1.25(0.71-2.22)$ & 0.440 & 1.56 (0.82-2.99) & 0.178 \\
\hline - Homozygous MT & $28(13.3 \%)$ & $7(9.9 \%)$ & $0.79(0.29-1.89)$ & 0.610 & $1.09(0.38-2.84)$ & 0.868 \\
\hline \multicolumn{7}{|l|}{ FLG 3321delA } \\
\hline -WT & 196 (93.3\%) & $64(90.1 \%)$ & Reference & & Reference & \\
\hline - Heterozygous MT & $14(6.7 \%)$ & $7(9.9 \%)$ & $1.53(0.56-3.85)$ & 0.379 & $1.78(0.61-4.81)$ & 0.265 \\
\hline \multicolumn{7}{|l|}{ FLGK4022X } \\
\hline$-W T$ & 195 (92.9\%) & $67(94.4 \%)$ & Reference & & Reference & \\
\hline - Heterozygous MT & $15(7.1 \%)$ & $4(5.6 \%)$ & $0.76(0.21-2.19)$ & 0.643 & $0.79(0.19-2.68)$ & 0.723 \\
\hline \multicolumn{7}{|l|}{$D F B 1$ C $2266 \mathrm{~T}$} \\
\hline$-W I$ & $50(22.8 \%)$ & (14) & Therencence & & 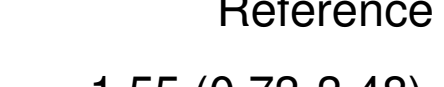 & \\
\hline - Helerozygous IMI & $121(57.6 \%)$ & 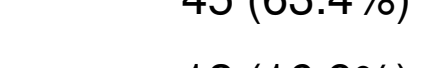 & $1.33(0.68-2.7)$ & 0.416 & $1.53(0.13 / 3-3.48)$ & 0.270 \\
\hline \multirow{2}{*}{\multicolumn{7}{|c|}{$K D R$}} \\
\hline & & & & & & \\
\hline -WT & $164(78.1 \%)$ & 56 (78.9\%) & Reference & & Reference & \\
\hline \multicolumn{7}{|l|}{ ILSRA } \\
\hline -WT & $89(42.4 \%)$ & $37(52.1 \%)$ & Reference & & Reference & \\
\hline - Heterozygous MT & $121(57.6 \%)$ & $34(47.9 \%)$ & $0.68(0.39-1.16)$ & 0.155 & $0.73(0.40-1.33)$ & 0.306 \\
\hline \multicolumn{7}{|l|}{ LQ } \\
\hline -wT & $147(70.0 \%)$ & $44(62.0 \%)$ & Reference & & Reference & \\
\hline - Heterozygous MT & $62(29.5 \%)$ & $26(36.6 \%)$ & $1.40(0.79-2.46)$ & 0.245 & $1.66(0.88-3.15)$ & 0.117 \\
\hline - Homozygous MT & $1(0.5 \%)$ & $1(1.4 \%)$ & $3.34(0.13-85.65)$ & 0.400 & $5.38(0.17-174.84)$ & 0.294 \\
\hline \multicolumn{7}{|l|}{ IL12RB1 a } \\
\hline -WT & 105 (50.0\%) & $38(53.5 \%)$ & Reference & & Reference & \\
\hline \multirow{2}{*}{\multicolumn{7}{|c|}{ 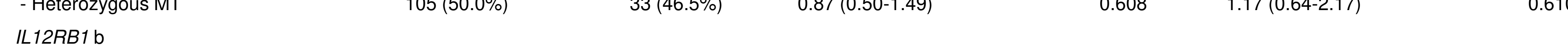 }} \\
\hline & & $52(73.0 \%)$ & Reforence & & Reforence & \\
\hline - Heterozygous MT & $81(38.6 \%)$ & $16(22.5 \%)$ & $0.47(0.25-0.87)$ & 0.019 & $0.60(0.29-1.17)$ & 0.141 \\
\hline - Homozygous MT & $5(2.4 \%)$ & $3(4.2 \%)$ & $1.43(0.29-6.05)$ & 0.480 & 1.78 (0.33-8.19) & 0.464 \\
\hline
\end{tabular}

- The heterozygous mutation of IL12RB1b showed lower odds of developing concurrent ACD in AD patients. But the difference was diminished after adjusting for age and sex

- Overall, there was no genetic difference between 'AD only' and 'AD+ACD'

\section{CONCLUSION}

$25.3 \%$ of AD patients had concurrent ACD,

suggesting increased contact sensitization in AD patients compared to normal population.

However, this study had failed to find any genetic differences

between the AD patients with and without ACD.

Patients with $A D$ who fall into one of the following cases should be

strongly suspected for concurrent ACD and patch testing should be done

- The patients with self-reported family history of ACD

- In absence of family history of ACD

- Male over 20 years old

- Female over 14 years old

The followings may suggest the possibility of concurrent ACD and patch testing may be considered

- The patients with self-reported personal history of ACD

- Adolescent or adult-onset disease

- The presence of prurigo nodularis

\section{ACKNOWLEDGEMENTS}

This research was supported by a grant of the Korea Healthcare Technology R\&D Project through the Korean Health Industry Development Institute (KHIDI),

funded by the Ministry of Health \& Welfare, Republic of Korea (grant no. HI14C2687) 